\title{
МОЛЕКУЛЯРНО-ГЕНЕТИЧЕСКИЕ И ФЕНОТИПИЧЕСКИЕ ОСОБЕННОСТИ СЛУЧАЕВ ВОЗНИКНОВЕНИЯ ДЕСМОИДНОГО ФИБРОМАТОЗА
}

\author{
Т. А. Музаффрарова', О. В. Новикова², И. Ю. Сачков ${ }^{3}$, Ф. М. Кипкеева ${ }^{1}$, Е. К. Гинтер ${ }^{1}$, А. В. Карпухин ${ }^{1}$ \\ ${ }^{1}$ Медико-генетический научный центр имени Н. П. Бочкова, Москва, Россия \\ ${ }^{2}$ Московский научно-исследовательский онкологический институт имени П. А. Герцена, Москва, Россия \\ ${ }^{3}$ Государственный научный центр колопроктологии имени А. Н. Рыжих, Москва, Россия
}

\begin{abstract}
Десмоидные фибромы (ДФ) - редкие мезенхимальные опухоли с частотой возникновения 2-4 случая на 1 млн человек в год. Они могут возникать как спорадически, так и в ассоциации с семейным аденоматозным полипозом (САП). Природа возникновения спорадических ДФ ранее не была выяснена. Целью исследования было определить возможную значимость герминальных мутаций гена АРС у пациентов со спорадическими ДФ. Экзоны гена APC амплифицировали и исследовали с помощью конформационно-чувствительного электрофореза в полиакриламидном геле и последующего секвенирования по Сэнгеру. Статистическую обработку результатов проводили с помощью пакета программ «Statistica 10». При исследовании 51 случая спорадических ДФ мутации выявлены у 6 человек (12\%). Пациенты с выявленными мутациями имели характерный фенотип: раннюю манифестацию (в среднем в 5,8 года, в то время как у пациентов без мутаций - в 19 лет ( $p=0,02)$ ); тяжелое течение заболевания; мультифокальный рост ДФ, локализованных на туловище, и неблагоприятный прогноз. Все выявленные мутации были обнаружены в области 3'-конца гена АРС. Для сравнения со спорадическими были исследованы ДФ, связанные с САП (12 человек), мутации выявлены у 6 из них. При мутации в гене АРС у пациентов с САП не было выявлено случаев множественных ДФ, фибромы у пациентов с САП развивались позже (35 лет), чем у пациентов со спорадическими ДФ ( $\rho$ = 0,004), Следовательно, при мутациях в одном и том же гене фенотипы спорадических и ДФ, связанных с САП, различны. Для спорадического ДФ характерно более частое расположение мутаций на З'-конце гена АРС по сравнению с ДФ при САП. Таким образом, впервые среди спорадических ДФ охарактеризован подтип с фенотипическими особенностями, обусловленными герминальными мутациями в гене АРС
\end{abstract}

Ключевые слова: спорадический десмоидный фиброматоз, ген АРС, мультифокальные десмоидные опухоли, семейный аденоматозный полипоз

Информация о вкладе авторов: Т. А. Музаффарова - проведение исследования, работа с литературными данными, оформление рукописи О. В. Новикова, И. Ю. Сачков - предоставление образцов и клинических данных пациентов; Ф. М. Кипкеева, Е. К. Гинтер - работа с литературными данными, участие в оформлении рукописи; А. В. Карпухин — организация исследования, оформление рукописи.

Соблюдение этических стандартов: исследование одобрено комитетом по этике ФГБНУ «МГНЦ имени Бочкова Н. П.» (протокол № 3 от 09 февраля 2012 г.)

$\triangle$ Для корреспонденции: Фатима Магомедовна Кипкеева ул. Москворечье, д. 1, г. Москва, 115522; BRCA1@mail.ru

Статья получена: 25.06.2019 Статья принята к печати: 13.07.2019 Опубликована онлайн: 18.07.2019

DOI: 10.24075/vrgmu.2019.045

\section{MOLECULAR-GENETIC AND PHENOTYPIC CHARACTERISTICS OF DESMOID-TYPE FIBROMATOSIS}

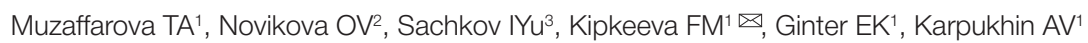

${ }^{1}$ Bochkov Research Center for Medical Genetics, Moscow, Russia

${ }^{2}$ Hertsen Moscow Oncology Research Center, Moscow, Russia

${ }^{3}$ Ryzhikh State Research Center for Coloproctology, Moscow, Russia

Desmoid-type fibromatosis (DF) is a rare mesenchymal tumor occurring in only 2 to 4 people per 1,000,000 population a year. Desmoid tumors are either seen sporadically or in individuals with familial adenomatous polyposis (FAP). The etiology of sporadic DF is uncertain. The aim of this study was to estimate the potential significance of germline mutations in the APC gene in patients with sporadic DF. APC exons were amplified, studied using conformation sensitive gel electrophoresis and then Sanger-sequenced. The obtained data were processed in Statistica 10. Mutations were detected in 6 (12\%) of 51 participants with sporadic DF. Those 6 patients shared a typical DF phenotype characterized by early age of onset ( 5.8 years on average, in contrast to the patients without $A P C$ mutations, who developed DF at 19 years of age; $p=0.02$ ), severe clinical course, multifocal localization on the trunk, and poor prognosis. All of the detected $A P C$ mutations were localized to the $3^{\prime}$-end of the gene. For the purpose of comparison, we analyzed a sample of 12 patients with FAP-associated DF. Of those patients, 6 carried mutations in the APC gene. In the analyzed sample, the patients with FAP and the mutant APC gene developed DF at older age ( 35 years) than the patients with sporadic DF $(p=0.004)$ and their tumors were not multifocal. This means that sporadic and FAP-associated desmoids have different phenotypes in patients with $A P C$ mutations. Patients with sporadic tumors have mutations at the 3 '-end of the APC gene more often than individuals with FAP-associated DF. To our knowledge, this is the first study to characterize the subtype of sporadic desmoid fibromatosis phenotypically determined by germline mutations in the $A P C$ gene.

Keywords: sporadic desmoid-type fibromatosis, APC gene, multifocal desmoid tumors, familial adenomatous polyposis

Author contribution: Muzaffarova TA conducted the study, analyzed the literature and wrote the manuscript; Novikova OV, Sachkov IYu provided patients' samples and medical histories; Kipkeeva FM, Ginter EK analyzed the literature and wrote the manuscript; Karpukhin AV organized the study and revised the manuscript.

Compliance with ethical standards: the study was approved by the Ethics committee of Bochkov Research Center for Medical Genetics (Protocol № 3 dated February 09, 2012).

$\triangle$ Correspondence should be addressed: Fatima M. Kipkeeva Moskvorechie 1, Moscow, 115522; BRCA1@mail.ru

Received: 25.06.2019 Accepted: 13.07.2019 Published online: 18.07.2019

DOI: $10.24075 /$ brsmu.2019.045

Десмоидные фибромы (ДФ) - гетерогенные доброкачественные опухоли, возникающие из глубоких мышечно-апоневротических структур. Они инфильтрируют окружающие мягкие ткани, но не метастазируют. ДФ состоят из веретеновидных клеток (фиброцитоподобных) и избыточного количества коллагеновых волокон. Опухоль лишена капсулы и по периферии глубоко проникает в виде тяжей между мышечными волокнами, приводя к их атрофии. Помимо этого, десмоиды способны распространяться на значительные расстояния от основной опухоли 
в виде узких тяжей, длина которых иногда достигает 20-30 cм.

ДФ могут развиваться практически в любой анатомической части тела. В зависимости от локализации их подразделяют на экстраабдоминальные (брюшная стенка, грудная клетка, конечности, шея, область малого таза) и интраабдоминальные (брыжейка, забрюшинное пространство). В связи с тем, что ДФ не метастазируют, формально их следовало бы отнести к доброкачественным новообразованиям. Но из-за присущего им агрессивного роста и склонности к многократным рецидивам после хирургического лечения они более соответствуют злокачественным опухолям [1].

ДФ могут достигать огромных размеров и при ряде локализаций приводят к летальному исходу. Частота ДФ в популяции составляет 2-4 случая на 1 млн человек в год [2]. Эффективное лечение ДФ остается сложной клинической проблемой из-за инфильтративного роста и локального агрессивного поведения. Традиционно методом выбора является хирургический. Однако частота рецидивов после операций варьирует от 45 до $90 \%$ [3].

Десмоидные опухоли возникают спорадически, а также могут быть связанными с семейным аденоматозным полипозом (САП) - наследственным заболеванием толстого кишечника, приводящим к развитию рака толстой кишки. Наибольшая доля случаев возникновения САП является следствием мутаций в гене, отвечающем за развитие полипоза толстого кишечника, или АРС (adenomatous polyposis coli gene). В 10-15\% случаев у пациентов с САП обнаруживают ДФ. Риск развития ДФ у пациентов с САП составляет 2,56 случаев на 1000 пациентов в год, что в 852 раза выше, чем в общей популяции [2]. В отличие от спорадических ДФ, САП-ассоциированные ДФ в 80\% случаев интраабдоминальные. Десмоидный фиброматоз преимущественно встречается у женщин и может манифестировать в любом возрасте, но чаще ДФ развиваются в возрасте 30-40 лет. В большинстве случаев САП-ассоциированные ДФ возникают в течение 5 лет после перенесенной операции [4].

Природа возникновения спорадических ДФ неясна. У пациентов с этим заболеванием были описаны случаи выявления соматических мутаций в гене $A P C$, но чаще всего такие мутации обнаруживают в гене $\beta$-катенина [5-6], который задействован в Wnt-сигнальном пути. Мутации в гене CTNNB1 (ген $\beta$-катенина) приводят к накоплению продукта этого гена в ядре фибробластов, что, в свою очередь, нарушает путь дифференцировки клетки и межклеточные взаимосвязи [7].
Генетические причины возникновения ДФ и их связь с клиническими проявлениями этого заболевания в настоящее время недостаточно изучены. Поскольку ДФ с высокой частотой возникают при САП, естественным кандидатом, обусловливающим предрасположенность и к спорадическим случаям ДФ, выступает ген АРC. Однако данные мировой литературы по исследованию герминальных мутаций в гене APC при спорадических случаях ДФ пока немногочисленны [8, 9].

Целью работы было изучить выборку пациентов со спорадическими случаями ДФ, не имевших признаков аденоматозного полипоза и родственников, страдающих САП и ДФ, и определить возможную значимость герминальных мутаций гена $A P C$ у таких пациентов. Для сравнения было решено отдельно исследовать молекулярные характеристики гена APC среди пациентов с ДФ при САП.

\section{ПАЦИЕНТЫ И МЕТОДЬ}

Исследование проводили в лаборатории молекулярной генетики СНЗ ФГБНУ «МГНЦ им. Н. П. Бочкова» с 2012 по 2017 г. Изучали две выборки пациентов. Первая выборка состояла из 51 человека с ДФ (21 мужчина, 30 женщин) в возрасте от месяца до 60 лет (см. рис.); медиана составила 16,8 лет. Образцы крови получили от пациентов, обратившихся в МНИОИ им. П. А. Герцена филиал ФГБУ «НМИЦ радиологии» Минздрава России. Критерии включения пациентов в исследование: наличие основного диагноза ДФ; отсутствие на момент проведения исследования характерных жалоб со стороны желудочно-кишечного тракта, позволяющих заподозрить диффузный полипоз толстой кишки; отсутствие семейной истории САП; отсутствие случаев возникновения ДФ у родственников. Таким образом, все случаи ДФ в этой выборке рассматривали как спорадические.

Десмоидные опухоли имели различную локализацию (на спине, в области грудной и брюшной стенок, на конечностях, интраабдоминально). У 11 пациентов наблюдали мультифокальный рост ДФ (см. раздел «Результаты исследования»). У части пациентов ДФ рецидивировали.

При исследовании 65 пациентов с САП (образцы крови получили из Государственного научного центра колопроктологии им. А. Н. Рыжих) была сформирована вторая выборка, в которую вошли 12 человек: 2 мужчин и 10 женщин. Возраст возникновения ДФ у больных варьировал от 24 до 57 лет; медиана составила 32,5 лет. Критерии включения в исследование: наличие полипоза

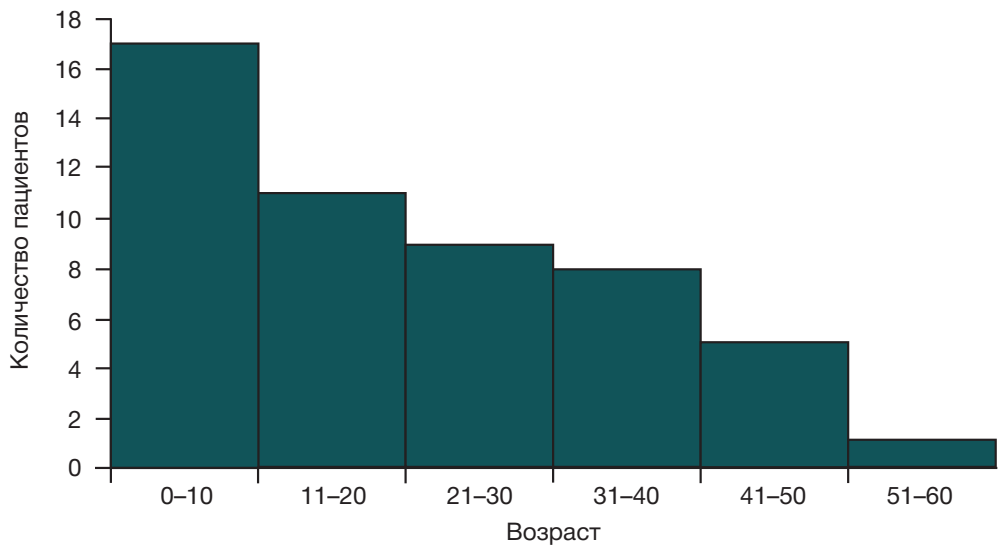

Рис. Распределение больных со спорадическими ДФ по возрасту 
толстой кишки; наличие ДФ. Наследственность по САП отягощена у 8 человек. У четверых пациентов семейная история не прослежена. Все ДФ возникли после проведения оперативного вмешательства и были локализованы на передней брюшной стенке или интраабдоминально, преимущественно являлись единичными

Определение мутаций в гене АPC проводили в ДНК, выделенной из периферической крови больных. ДНК получали из лейкоцитов периферической крови стандартным фенол-хлороформным методом [10] Анализ гена $A P C$ на наличие мутаций проводили согласно литературным данным [11]. Кодирующие экзоны гена APC амплифицировали при помощи экзонспецифичных праймеров. ПЦР-продукты исследовали конформационно-чувствительным электрофорезом в полиакриламидном геле (окрашивание серебром). Изменение первичной структуры гена дополнительно исследовали секвенированием по Сэнгеру с использованием набора Big DyeTM Terminator v. 3.1 Cycle Sequencing и ДНК-анализатора ABI Prism 3130x1 (Applied Biosystems; США). Хроматограммы интерпретировали при помощи ChromasPro и интернетресурсов NCBI BLAST и Ensembl genome browser 91. В качестве образца сравнения использовали референсную последовательность гена APC NM_000038.6.

Статистическую обработку результатов проводили с помощью пакета программ Statistica 10.0 (StatSoft; USA).

\section{РЕЗУЛЬТАТЫ ИССЛЕДОВАНИЯ}

Молекулярный анализ гена АPC проводили у 51 пациента со спорадическими ДФ. В 6 случаях (12\%) выявлены герминальные мутации в гене АPC. Обнаруженные мутации в гене APC, возраст пациентов на момент постановки диагноза, количество (единичные, мультифокальные) и локализация ДФ представлены в табл. 1

Из 6 выявленных нами мутаций 2 мутации являются новыми: с.4386-4390 delGAGAG (1462delGAGAG) и c.4575insT (1525insT). Обе указанные новые мутации приводят к сдвигу рамки считывания с преждевременным образованием стоп-кодона и, следовательно, являются патогенными.

Мутация с.4575insТ обнаружена у пациентки № 1 (табл. 1). По клиническим данным отмечали тяжелое течение ДФ с ранней манифестацией (9 лет) и мультифокальным ростом ДФ. В возрасте 19 лет при эндоскопическом обследовании толстой кишки полипоз не выявлен.

Пациент № 2 имеет мутацию с.4386-4390 delGAGAG (табл. 1). Манифестация заболевания у него была отмечена в возрасте 1 месяц. С раннего детства наблюдали мультифокальный рост ДФ грудной стенки. K возрасту 18 лет пациент перенес 5 операций, курсы химиотерапии, гормональной и лучевой терапии без стабилизации процесса. Таким образом, заболевание можно охарактеризовать крайне тяжелым течением и устойчивостью к терапии. Мутация с.4386-4390 delGAGAG (1462delGAGAG) не выявлена в других популяциях [12-14]. Мутация возникла de novo, так как в крови матери и отца пациента не была обнаружена. Чувствительность использованного метода определения мутаций позволяет выявить 1-5\% мутантных аллелей, что указывает на малую вероятность мозаицизма у одного из родителей [15].

У 3 пациентов, не являющихся родственниками, была обнаружена одна и та же мутация - c.4393-4394 delAG (1465delAG). Несмотря на идентичность нарушения на уровне ДНК, клиническая картина имела различия. Среди этих пациентов - молодая женщина (28 лет) с дебютом заболевания в 17 лет. Отмечалось мультифокальное поражение грудной и брюшной стенок и интраабдоминальный рост ДФ. Признаков полипоза толстой кишки к возрасту 28 лет по результатам колоноскопии не обнаружено. Ту же самую мутацию имели еще 2 пациента: мальчик, у которого в области грудной стенки и поясницы были обнаружены множественные десмоиды при рождении, и девочка, у которой в возрасте 2 лет на спине развилась единичная ДФ. Обращает на себя внимание, что при одной мутации возраст начала заболевания, локализация и количество ДФ у всех 3 больных были различными. Различия в локализации ДФ, возможно, обусловлены разницей в возрасте больных, и, по мере взросления, не исключена вероятность развития мультифокального поражения грудной и брюшной стенок у всех больных с этой мутацией. Не исключено также, что на течение заболевания, обусловленного одной и той же мутацией, оказывают влияние факторы окружающей среды и различие генотипов больных.

У пациента с мутацией c.4348C/T; p.R1450X был отмечен мультисокальный рост ДФ с 15-летнего возраста. После неоднократного хирургического лечения и курсов химио- и гормональной терапии была достигнута стабилизация процесса.

У всех 6 пациентов этой группы была отмечена ранняя манифестация заболевания. Основное число больных (5 из 6) имели характерный фенотип в виде множественного роста узлов опухоли, плохо поддающихся лечению. Несмотря на проводившуюся терапию у 3 пациентов группы (50\%) прогно3 был неблагоприятным. Из 6 пациентов с мутацией в гене APC у 3 наблюдали тяжелое течение заболевания, что чаще, чем среди пациентов без герминальной мутации в этом гене (2 из 45). Это различие статистически значимо $(p=0,01)$.

Возраст возникновения ДФ среди пациентов с мутацией в гене $A P C$ варьировал от месяца до 17 лет (табл. 1); медиана составила 5,8 года. В то же время у пациентов без мутаций этот возраст варьировал от 1 месяца до 60 лет (см. рис. 1); медиана - 19 лет. Различие значений медиан статистически значимо ( $p=0,022 ; U$-тест).

Таблица 1. Мутации в гене АРC у пациентов со спорадическим десмоидным фиброматозом

\begin{tabular}{|c|l|l|c|}
\hline \multicolumn{1}{|c|}{ № } & \multicolumn{1}{|c|}{ Локализация ДФ } & \multicolumn{1}{|c|}{ Наименование мутации в гене APC } & \multicolumn{1}{|c|}{ Возраст возникновения первой ДФ } \\
\hline 1 & Мультифокальный рост, грудная и брюшная стенки & с.4575insT (1525insT) & лет \\
\hline 2 & Мультифокальный рост, грудная и брюшная стенки & с.4386-4390 delGAGAG (1462delGAGAG) & 2 месяца \\
\hline 3 & Мультифокальный рост, грудная стенка, поясничная область & R1450X (с.4348C/T) & 15 лет \\
\hline 4 & $\begin{array}{l}\text { Мультифокальный рост, грудная и брюшная стенки, } \\
\text { интраабдоминально }\end{array}$ & с.4393-4394 delAG (1465delAG) & 17 лет \\
\hline 5 & Мультифокальный рост, грудная стенка, поясничная область & с.4393-4394 delAG (1465delAG) & 1 месяц \\
\hline 6 & На спине & с.4393-4394 delAG (1465delAG) & 2 года \\
\hline
\end{tabular}


Все мутации в гене APC были обнаружены у больных с локализацией ДФ на туловище (6 пациентов с мутацией из 26 пациентов с локализацией ДФ на туловище, что составляет 23\%) и отсутствовали при других локализациях ДФ (табл. 2). Частота мутаций в гене APC при локализации опухоли на туловище значимо выше, чем при других локализациях ДФ ( $p=0,023)$. Этот феномен не обусловлен преимущественным накоплением множественных ДФ на туловище в целом (что могло бы указывать на случайное накопление при мутациях), так как их частота на туловище не была значимо выше по отношению к другим локализациям ДФ (табл. 2; $p=0,17$ ).

Из 51 пациента множественные ДФ имели 11 человек. У пяти из них обнаружены герминальные мутации в гене APC, что составило 45\% (табл. 2). В группе пациентов без мультифокального роста ДФ (40 человек) был обнаружен только один случай с мутацией в гене $A P C$ (1/40, или 2,5\%). Различие этих частот статистически значимо ( $p=0,001)$. На ассоциацию мутаций с множественными ДФ указывает также значение отношения шансов (OR = 32,5; 95\% Cl: 3,22-326,31). Следовательно, мутации в этом гене преимущественно встречаются у пациентов с множественными ДФ.

Таким образом, мутации в гене АPC были связаны с локализацией ДФ на туловище и их преимущественно мультисфокальным ростом (табл. 2).

Столь значительное количество мутаций (12\%) среди спорадических случаев ДФ без признаков и (или) семейной истории САП ставит вопрос о возможном различии мутаций в гене АРC при САП и при спорадических ДФ. Все выявленные мутации в группе пациентов со спорадическими ДФ были локализованы на З'-конце от кодона 1444 гена АРС.

В связи с этим была изучена выборка пациентов с САП и ДФ. Из 65 пациентов с САП у 12 человек полипоз толстого кишечника сопровождали ДФ. Герминальные мутации в гене АРC были обнаружены у 6 человек из 12 пациентов с САП и ДФ (табл. 3).

Различий в клинической картине фиброматоза у пациентов с САП при мутациях и без мутаций в гене АРC выявлено не было. Возраст возникновения ДФ среди пациентов с САП и мутацией в гене АРС варьировал от 28 до 57 лет (табл. 3), медиана составила 35,5 года, что не отличается значимо от среднего значения у пациентов без мутаций - 29 лет (диапазон 24-36 лет).

Ни один пациент с САП и ДФ не имел мутацию в гене APC на 3'-конце от кодона 1444 гена APC (табл. 3) При спорадических случаях ДФ все найденные мутации располагались ближе к 3'-концу от кодона 1444 (табл. 1). Различие в локализации мутаций при спорадических и связанных с САП ДФ статистически значимо ( $p=0,0022$, $\mathrm{OR}=144 ;$ 95\% Cl: 2,43-8517,50), т. е. для спорадических случаев ДФ характерно более частое расположение мутаций на З'-конце гена АРС по сравнению с ДФ при САП.

Следует отметить более ранний возраст начала заболевания при мутациях в гене APC среди спорадических ДФ по отношению к ДФ при САП медианы 5,8 года и 35,5 года соответственно ( $p=0,004$; U-тест). Статистически значимых различий в возрасте манифестации заболевания у пациентов без мутаций при спорадических и ассоциированных с САП ДФ выявлено не было $(p=0,09)$.

Случаи мультифокального ДФ у больных с мутацией в гене APC чаще выявлены среди пациентов со спорадическим ДФ (5/6), нежели у пациентов с САП $(0 / 6)-\mathrm{OR}=60 ; 95 \% \mathrm{Cl}: 1,64-2187,79 ; p=0,015$.

\section{ОБСУЖДЕНИЕ РЕЗУЛЬТАТОВ}

В настоящей работе изучены герминальные мутации в гене APC у 51 пациента со спорадическим десмоидным фиброматозом. У всех пациентов выборки отсутствовали

Таблица 2. Связь мутаций в гене АРС с различной локализацией и количеством ДФ

\begin{tabular}{|l|c|c|c|c|}
\hline \multirow{2}{*}{ Локализация десмоидных опухолей } & \multicolumn{2}{|c|}{ Больные с десмоидным фиброматозом } & $\begin{array}{c}\text { Больные с десмоидным фиброматозом с развитием } \\
\text { мультифокальных опухолей }\end{array}$ \\
\cline { 2 - 5 } & Число пациентов & Из них с мутациями в гене АРС & Число пациентов & Из них с мутациями в гене АРС \\
\hline Грудная и/или брюшная стенка & 26 & 6 & 8 & - \\
\hline Интраабдоминально & 3 & - & - & - \\
\hline Конечности & 14 & - & - & - \\
\hline Другая локализация & 8 & - & 11 \\
\hline Всего & 51 & & - \\
\hline
\end{tabular}

Таблица 3. Характеристика мутаций в гене АРС и клинические показатели пациентов с ДФ при САП

\begin{tabular}{|c|c|c|c|}
\hline № & Наименование мутации & Характеристика ДФ & Возраст, годы \\
\hline 1 & c.3464-3468 delAAGAA (1155del5) & Десмоид корня брыжейки послеоперационный & 57 \\
\hline 2 & c.3927-3921delAAAGA (1309del5) & Десмоид в послеоперационном рубце & 28 \\
\hline 3 & c.3930insA (1310insA) & Десмоид корня брыжейки послеоперационный & 34 \\
\hline 4 & c.3183-3187 delACAAA (1061del5) & Десмоид корня брыжейки послеоперационный & 38 \\
\hline 5 & c.2274-2278 delAGCCC p.K758Nfs (758-760 delAGCCC) & Десмоид брюшной стенки послеоперационный & 30 \\
\hline 6 & 3496delT (1166delT) & Десмоид брюшной стенки & 33 \\
\hline 7 & - & Десмоид брюшной стенки послеоперационный & 33 \\
\hline 8 & - & Десмоид корня брыжейки послеоперационный & 29 \\
\hline 9 & - & Десмоид корня брыжейки послеоперационный & 29 \\
\hline 10 & - & Десмоид брюшной стенки послеоперационный & 29 \\
\hline 11 & - & Десмоид брюшной стенки & 24 \\
\hline 12 & - & Десмоид брюшной стенки послеоперационный & 36 \\
\hline
\end{tabular}


симптомы и семейный анамнез САП. У 6 человек (12\%) из этой выборки впервые были найдены патогенные мутации, две из которых (1525insT и 1462delGAGAG) до настоящего времени не были описаны. Ранее мутации в гене APC анализировали на выборках, включавших как спорадические ДФ, так и ассоциированные с САП. Мутации были выявлены только у больных с САП; среди пациентов со спорадическим ДФ мутации не были обнаружены. Это может быть связано как с задачами исследования, так и с характерными особенностями выборки. Так, в одном из исследований присутствовал только один случай множественных ДФ [16].

Ранняя манифестация десмоидного фиброматоза встречается во врачебной практике очень редко и в литературе ее обсуждают в основном как клинический случай [17, 18]. В нашей выборке пациентов со спорадическими ДФ и мутацией в гене АРC зарегистрировано 3 случая с ранней манифестацией заболевания (двум пациентам диагноз поставлен в возрасте 1 и 2 месяцев, и одному ребенку - в 2 года).

Все мутации в гене АРС у больных со спорадическими ДФ были локализованы на 3'-конце от кодона 1444 и ассоциированы с тяжелым течением заболевания в виде мультифокальных ДФ и ранней манифестации (медиана возраста начала заболевания составила 5,8 года, в отличие от значения, полученного среди больных ДФ без мутаций, и составившего 19 лет). Также все ДФ у пациентов с мутацией в АРС были локализованы на туловище, хотя в целом при десмоидном фиброматозе локализация на туловище не была значимо выше, чем другие локализации (см. табл. 2; $p=0,17)$. Эти наши данные получены впервые. В литературе описаны семьи с наследственной десмоидной болезнью. Например, есть сообщение о семье, где в трех поколениях родственники наследуют десмоидные опухоли. Десмоидный фиброматоз у них связан с мутацией сдвига рамки считывания в кодоне 1924 гена АРC. У больных членов семьи наблюдаются множественные ДФ различной локализации как экстраабдоминальные, так и интраабдоминальные. Возраст возникновения ДФ варьирует от рождения до 10-20 лет жизни. Из 9 больных ДФ в этой семье полипоз либо рак толстой кишки был зафиксирован у троих человек. Другие немногочисленные случаи описания семей с наследственным десмоидным фиброматозом также связаны с мутациями в гене APC на 3'-конце от кодона 1444, характеризуются возникновением множественных ДФ и тяжелым течением заболевания [19, 20].

Также было интересно сравнить выявленные характеристики мутаций и фенотипические особенности спорадических ДФ с таковыми при САП. Данные о связи генотипа с клинической картиной САП, полученные в разных работах, различаются. Так, в одной работе связи с локализацией мутации в гене $A P C$ и развитием ДФ у больных с САП выявлено не было [21], в другой из 14 пациентов с САП и ДФ только у двоих мутация была расположена на 3'-конце от кодона 1444 [22], что может быть обусловлено популяционными особенностями. Была изучена российская выборка САП. Среди 65 больных с САП 12 имели ДФ (18\%). Данное значение близко к значениям, полученным в других работах: по одним данным оно составило 3,5-32\% [23], по другим - 10-15\% [24]. У половины пациентов с САП и ДФ из нашей выборки были обнаружены мутации в гене АPC. Все найденные 6 мутаций были локализованы на 5'-конце от кодона 1444. Различие в частотах расположения мутаций относительно кодона 1444 при спорадических ДФ и ассоциированных с САП статистически значимо ( $p=0,002)$. Мы сравнили частоты мутаций относительно кодона 1444 при ДФ, ассоциированных с САП, полученные в нашей работе с аналогичными частотами, полученными на объединенной выборке больных с САП из нескольких стран [25]. Указанные частоты статистически значимо не различались $(p=0,34)$. В то же время, полученные нами частоты расположения мутаций относительно кодона 1444 при спорадических ДФ отличались также от частот при ДФ, ассоциированных с САП, полученных другими исследователями (p=0,0002) [25]. Следовательно, спорадические ДФ обусловлены мутациями, локализованными на 3'-конце от 1444 кодона, чаще, чем десмоидные опухоли, ассоциированные с САП.

У пациентов с САП в нашей выборке первичных ДФ обнаружено не было: все десмоидные опухоли возникли после операции и были локализованы внутрибрюшинно либо на брюшной стенке. Такие же особенности течения ДФ у пациентов с САП были отмечены и в других исследованиях [26, 27].

Спорадические ДФ при мутациях в гене АРC были преимущественно мультифокальными и локализованы в области грудной и (или) брюшной стенки, Интраабдоминальная локализация ДФ характерна для САП и существенно реже встречается при спорадических ДФ [25]. Вероятнее всего, абдоминальная локализация ДФ при САП обусловлена травмированием тканей при проведении оперативного вмешательства на органах брюшной полости [28]. Напротив, при спорадических ДФ с мутациями в гене АРС прямой связи с травмой не наблюдалось.

Медианы возраста развития ДФ у больных с мутациями и без мутаций не отличались в нашей выборке с САП, в то время как возраст манифестации заболевания при спорадических ДФ у больных с герминальными мутациями в АРС был существенно ниже. Следует отметить, что возрасты диагноза спорадических и САП-ассоциированных ДФ у пациентов без мутаций статистически значимо не различались $(p=0,09)$.

Эти данные указывают на существенные различия клинических картин развития спорадических ДФ и ДФ, ассоциированных с САП, среди больных с мутацией в гене APC, связанные, по крайней мере частично, с положением мутации в этом гене.

Таким образом, в выборке больных со спорадическими ДФ выявлена группа больных с особенностями течения заболевания, обусловленными герминальной мутацией в гене APC. Для таких пациентов характерны: мультифокальный рост ДФ, ранняя манифестация, тяжелое течение, неэффективность проводимой терапии и, как следствие, неблагоприятный прогноз заболевания. Существуют молекулярные особенности, связанные с вышеописанной клинической картиной. Мутация в этих случаях локализована на 3'-конце гена $A P C$

Полученная информация существенна для выбора тактики лечения таких больных и мер профилактики полипоза, а также открывает возможности изучения механизмов, приводящих к развитию мультифокальных ДФ, но не САП.

\section{ВЫВОДЫ}

Среди больных с ДФ без семейной истории аденоматозного полипоза обнаружен подтип с герминальными мутациями в гене APC и фенотипическими особенностями. Больные 
с мутациями имеют фенотип, отличающий их от больных с ДФ без мутаций, - более ранний возраст возникновения ДФ, как правило, множественных и располагающихся на туловище. По этим характеристикам больные со спорадическими ДФ с мутациями отличаются и от больных с ДФ при САП, также с герминальными мутациями в гене АРС. При этом для спорадических ДФ характерно более частое расположение мутаций на 3'-конце гена APC. Полученные результаты следует учитывать при лечении больных с ДФ и разработке подходов к профилактике как ДФ, так и САП. Они также создают возможности для исследования молекулярных механизмов, приводящих в результате мутаций в гене АРC к первичному возникновению ДФ, а не САП.

\section{Литература}

1. Глебовская В. В. Терморадиотерапия больных с первичным и рецидивным экстраабдоминальным десмоидом [диссертация]. M., 2004.

2. Eastley N, McCulloch T, Esler C, Hennig I, Fairbairn J, Gronchi A, et al. Extra-abdominal desmoid fibromatosis: A review of management, current guidance and unanswered questions. Eur J Surg Oncol. 2016; 42 (7): 1071-83. DOI: 10.1016/j. ejso.2016.02.012.

3. Ткачев С. И., Алиев М. Д., Глебовская В. В. и др. Применение терморадиотерапии у больных первичными и рецидивными зкстраабдоминальными десмоидными опухолями. Саркомь костей, мягких тканей и опухоли кожи. 2009; (1): 34-7.

4. DE Marchis ML, Tonelli F, Quaresmini D, Lovero D, Della-Morte D, Silvestris $\mathrm{F}$, et al. Desmoid Tumors in Familial Adenomatous Polyposis. Anticancer Res. 2017 Jul; 37 (7): 3357-66.

5. Mullen JT, DeLaney TF, Rosenberg AE, Le L, lafrate AJ, Kobayashi W, et al. $\beta$-Catenin mutation status and outcomes in sporadic desmoid tumors. Oncologist. 2013; 18 (9): 1043-9. DOI: 10.1634/ theoncologist. 2012-0449.

6. Alman BA, Li C, Pajerski ME, Diaz-Cano S, Wolfe HJ. Increased beta-catenin protein and somatic APC mutations in sporadic aggressive fibromatoses (desmoid tumors). Am J Pathol. 1997; 151 (2): 329-34. PubMed PMID: 9250146; PubMed Central PMCID: PMC1857985.

7. Никулин М. П., Петросян А. П., Цымжитова Н. Ц., Губина Г. И. Забрюшинные десмоиды: аналитический обзор и случай из практики. Клиническая и экспериментальная хирургия. 2015; (4): 103-12.

8. Koskenvuo L, Peltomäki P, Renkonen-Sinisalo L, Gylling A, Nieminen TT, Ristimäki A, et al. Desmoid tumor patients carry an elevated risk of familial adenomatous polyposis. J Surg Oncol. 2016; 113 (2): 209-12. DOI: 10.1002/jso.24117.

9. Brueckl WM, Ballhausen WG, Förtsch T, Günther K, Fiedler W, Gentner B, et al. Genetic testing for germline mutations of the APC gene in patients with apparently sporadic desmoid tumors but a family history of colorectal carcinoma. Dis Colon Rectum. 2005; 48 (6): 1275-81. DOI: 10.1007/s10350-004-0949-5.

10. Sambrook J, Fritsch EF, Maniatis T. Molecular cloning: a laboratory manual, N.Y.: Cold Spring Harbor Laboratory, Cold Spring Harbor, 1989; $1546 \mathrm{p}$.

11. Музаффрарова Т. А., Мансорунов Д. Ж., Сачков И. Ю., Кузеванова А. Ю., Карпухин А. В., Алимов А. А. Молекулярногенетические аспекты риска семейного аденоматозного полипоза. Молекулярная медицина. 2018; 16 (6): 60-4. DOI: https://doi.org/10.29296/24999490-2018-06-11.

12. The Human Gene Mutation Database $\left(H_{G M D}{ }^{\oplus}\right)$. Available from: http://www.hgmd.cf.ac.uk/ac/index.php.

13. Ensembl Genome Browser 96. Available from: http://www ensembl.org/index.html.

14. LOVD database. Available from: https://www.lovd.nl/.

15. Hes FJ, Nielsen M, Bik EC, Konvalinka D, Wijnen JT, Bakker E, et al. Somatic APC mosaicism: an underestimated cause of polyposis coli. Gut. 2008; 57 (1): 71-6. DOI: 10.1136/gut.2006.117796.

16. Kattentidt Mouravieva AA, Geurts-Giele IR, de Krijger RR,

van Noesel MM, van de Ven CP, van den Ouweland, et al. Identification of Familial Adenomatous Polyposis carriers among children with desmoid tumours. Eur J Cancer. 2012 Aug; 48 (12): 1867-74. DOI: 10.1016/j.ejca.2012.01.004

17. Dalit A, Karen M, Alexander M. Congenital desmoid tumor of the cheek: a clinicopathological case report. Eplasty. 2009 Nov 10; (9): e52. PubMed PMID: 20011031; PubMed Central PMCID: PMC2779781.

18. Roggli VL, Kim HS, Hawkins E. Congenital generalized fibromatosis with visceral involvement. A case report. Cancer. 1980; (45): 954-60.

19. Halling KC, Lazzaro CR, Honchel R, Bufill JA, Powell SM, Arndt CAS, et al. Hereditary Desmoid Disease in a Family with a Germline Alu IRepeat Mutation of the APC Gene. Hum Hered. 1999; (49): 97-102.DOI: 10.1159/000022852.

20. Eccles DM, van der Luijt R, Breukel C, Bullman H, Bunyan D, Fisher A, et al. Hereditary desmoid disease due to a frameshift mutation at codon 1924 of the APC gene. Am J Hum Genet. 1996; 59 (6): 1193-201. PMCID: PMC1914868; PMID: 8940264.

21. Nieuwenhuis MH, De Vos Tot Nederveen Cappel W, Botma A, Nagengast FM, Kleibeuker JH, Mathus-Vliegen EM, et al. Desmoid tumors in a dutch cohort of patients with familial adenomatous polyposis. Clin Gastroenterol Hepatol. 2008; 6 (2): 215-9. DOI: 10.1016/j.cgh.2007.11.011.

22. Torrezan GT, da Silva FC, Santos EM, Krepischi AC, Achatz MI, Aguiar S Jr, et al. Mutational spectrum of the APC and MUTYH genes and genotype-phenotype correlations in Brazilian FAP, AFAP, and MAP patients. Orphanet J Rare Dis. 2013; (8): 54. DOI: 10.1186/1750-1172-8-54.

23. Fallen $\mathrm{T}$, Wilson M, Morlan B, Lindor NM. Desmoid tumors - a characterization of patients seen at Mayo Clinic 1976-1999. Fam Cancer. 2006; 5 (2): 191-4. DOI: 10.1007/s10689-005-5959-5.

24. Lips DJ, Barker N, Clevers H, Hennipman A. The role of APC and beta-catenin in the aetiology of aggressive fibromatosis (desmoid tumors). Eur J Surg Oncol. 2009 Jan; 35 (1): 3-10. DOI: 10.1016/j.ejso.2008.07.003

25. Nieuwenhuis $M H$, Lefevre $J H$, Bülow $S$, Järvinen $H$, Bertario $L$, Kernéis S, et al. Family history, surgery, and APC mutation are risk factors for desmoid tumors in familial adenomatous polyposis: an international cohort study. Dis Colon Rectum. 2011; 54 (10): 1229-34.DOI: 10.1097/DCR.0b013e318227e4e8.

26. Nieuwenhuis $M H$, Lefevre $J H$, Bülow $S$, Järvinen $H$, Bertario $L$, Kernéis $\mathrm{S}$, et al. A nation-wide study comparing sporadic and familial adenomatous polyposis-related desmoid-type fibromatoses. Dis Colon Rectum. 2011 Oct; 54 (10): 1229-34. DOI: 10.1002/ijc.25664.

27. Koskenvuo L, Ristimäki A, Lepistö A. Comparison of sporadic and FAP-associated desmoid-type fibromatoses. J Surg Oncol. 2017 Nov; 116 (6): 716-21. DOI: 10.1002/jso.24699.

28. Nieuwenhuis MH, De Vos Tot Nederveen Cappel W, Botma A, Nagengast FM, Kleibeuker JH, Mathus-Vliegen EM, et al. Desmoid tumors in a dutch cohort of patients with familial adenomatous polyposis. Clin Gastroenterol Hepatol. 2008 Feb; 6 (2): 215-9. DOI: 10.1016/j.cgh.2007.11.011. 
1. Glebovskaja W. Thermoradiotherapy of patients with primary and recurrent extraabdominal desmoid [dissertation]. M., 2004

2. Eastley N, McCulloch T, Esler C, Hennig I, Fairbairn J, Gronchi A, et al. Extra-abdominal desmoid fibromatosis: A review of management, current guidance and unanswered questions. Eur J Surg Oncol. 2016; 42 (7): 1071-83. DOI: 10.1016/j. ejso.2016.02.012.

3. Tkashev SI, Aliev MD, Glebovskaja VV, et al. The use of thermoradiotherapy in patients with primary and recurrent spasticdominant desmoid tumors. Sarcomas of bones, soft tissues and skin tumors. 2009; (1): 34-7.

4. DE Marchis ML, Tonelli F, Quaresmini D, Lovero D, Della-Morte D, Silvestris $\mathrm{F}$, et al. Desmoid Tumors in Familial Adenomatous Polyposis. Anticancer Res. 2017 Jul; 37 (7): 3357-66.

5. Mullen JT, DeLaney TF, Rosenberg AE, Le L, lafrate AJ, Kobayashi W, et al. $\beta$-Catenin mutation status and outcomes in sporadic desmoid tumors. Oncologist. 2013; 18 (9): 1043-9. DOI: 10.1634/ theoncologist.2012-0449.

6. Alman BA, Li C, Pajerski ME, Diaz-Cano S, Wolfe HJ. Increased beta-catenin protein and somatic APC mutations in sporadic aggressive fibromatoses (desmoid tumors). Am J Pathol. 1997; 151 (2): 329-34. PubMed PMID: 9250146; PubMed Central PMCID: PMC1857985

7. Nikulin MP, Petrosyan AP, Tsymzhitova NTs, Gubina GI. Retroperitoneal desmoids: analytical review and case report. Clin Experiment Surg. 2015; (4): 103-12.

8. Koskenvuo L, Peltomäki P, Renkonen-Sinisalo L, Gylling A Nieminen TT, Ristimäki $A$, et al. Desmoid tumor patients carry an elevated risk of familial adenomatous polyposis. J Surg Oncol. 2016; 113 (2): 209-12. DOI: 10.1002/jso.24117.

9. Brueckl WM, Ballhausen WG, Förtsch T, Günther K, Fiedler W, Gentner B, et al. Genetic testing for germline mutations of the APC gene in patients with apparently sporadic desmoid tumors but a family history of colorectal carcinoma. Dis Colon Rectum. 2005; 48 (6): 1275-81. DOI: 10.1007/s10350-004-0949-5.

10. Sambrook J, Fritsch EF, Maniatis T. Molecular cloning: a laboratory manual, N.Y.: Cold Spring Harbor Laboratory, Cold Spring Harbor, 1989; $1546 \mathrm{p}$

11. Muzaffarova TA, Mansorunov DJ, Sachkov IY, Kuzevanova AY, Karpukhin AV, Alimov AA. Molecular genetic aspects of the risk for family adenomatous polyposis. Molecular medicine. 2018; 16 (6): 60-64.

12. The Human Gene Mutation Database (HGMD ${ }^{\circledR}$ ). Available from: http://www.hgmd.cf.ac.uk/ac/index.php.

13. Ensembl Genome Browser 96. Available from: http://www. ensembl.org/index.html.

14. LOVD database. Available from: https://www.lovd.nl/.

15. Hes FJ, Nielsen M, Bik EC, Konvalinka D, Wijnen JT, Bakker E, et al. Somatic APC mosaicism: an underestimated cause of polyposis coli. Gut. 2008; 57 (1): 71-6. DOI: 10.1136/gut.2006.117796.

16. Kattentidt Mouravieva AA, Geurts-Giele IR, de Krijger RR, van Noesel MM, van de Ven CP, van den Ouweland, et al. Identification of Familial Adenomatous Polyposis carriers among children with desmoid tumours. Eur J Cancer. 2012 Aug; 48 (12): 1867-74. DOI: 10.1016/j.ejca.2012.01.004

17. Dalit A, Karen M, Alexander M. Congenital desmoid tumor of the cheek: a clinicopathological case report. Eplasty. 2009 Nov 10; (9): e52. PubMed PMID: 20011031; PubMed Central PMCID: PMC2779781.

18. Roggli VL, Kim HS, Hawkins E. Congenital generalized fibromatosis with visceral involvement. A case report. Cancer. 1980; (45): 954-60.

19. Halling KC, Lazzaro CR, Honchel R, Bufill JA, Powell SM, Arndt CAS, et al. Hereditary Desmoid Disease in a Family with a Germline Alu IRepeat Mutation of the APC Gene. Hum Hered. 1999; (49): 97-102.DOI: 10.1159/000022852.

20. Eccles DM, van der Luijt R, Breukel C, Bullman H, Bunyan D, Fisher A, et al. Hereditary desmoid disease due to a frameshift mutation at codon 1924 of the APC gene. Am J Hum Genet. 1996; 59 (6): 1193-201. PMCID: PMC1914868; PMID: 8940264.

21. Nieuwenhuis $M H$, De Vos Tot Nederveen Cappel W, Botma A, Nagengast FM, Kleibeuker JH, Mathus-Vliegen EM, et al. Desmoid tumors in a dutch cohort of patients with familial adenomatous polyposis. Clin Gastroenterol Hepatol. 2008; 6 (2): 215-9. DOI: 10.1016/j.cgh.2007.11.011.

22. Torrezan GT, da Silva FC, Santos EM, Krepischi AC, Achatz Ml, Aguiar S Jr, et al. Mutational spectrum of the APC and MUTYH genes and genotype-phenotype correlations in Brazilian FAP, AFAP, and MAP patients. Orphanet J Rare Dis. 2013; (8): 54. DOI: 10.1186/1750-1172-8-54.

23. Fallen T, Wilson M, Morlan B, Lindor NM. Desmoid tumors - a characterization of patients seen at Mayo Clinic 1976-1999. Fam Cancer. 2006; 5 (2): 191-4. DOI: 10.1007/s10689-005-5959-5.

24. Lips DJ, Barker N, Clevers H, Hennipman A. The role of APC and beta-catenin in the aetiology of aggressive fibromatosis (desmoid tumors). Eur J Surg Oncol. 2009 Jan; 35 (1): 3-10. DOI: 10.1016/..ejso.2008.07.003

25. Nieuwenhuis $M H$, Lefevre $J H$, Bülow $S$, Järvinen $H$, Bertario $L$, Kernéis S, et al. Family history, surgery, and APC mutation are risk factors for desmoid tumors in familial adenomatous polyposis: an international cohort study. Dis Colon Rectum. 2011; 54 (10): 1229-34.DOI: 10.1097/DCR.0b013e318227e4e8.

26. Nieuwenhuis $M H$, Lefevre $J H$, Bülow $S$, Järvinen $H$, Bertario $L$, Kernéis $\mathrm{S}$, et al. A nation-wide study comparing sporadic and familial adenomatous polyposis-related desmoid-type fibromatoses. Dis Colon Rectum. 2011 Oct; 54 (10): 1229-34. DOI: 10.1002/ijc.25664.

27. Koskenvuo L, Ristimäki A, Lepistö A. Comparison of sporadic and FAP-associated desmoid-type fibromatoses. J Surg Oncol. 2017 Nov; 116 (6): 716-21. DOI: 10.1002/jso.24699.

28. Nieuwenhuis MH, De Vos Tot Nederveen Cappel W, Botma A, Nagengast FM, Kleibeuker JH, Mathus-Vliegen EM, et al. Desmoid tumors in a dutch cohort of patients with familial adenomatous polyposis. Clin Gastroenterol Hepatol. 2008 Feb; 6 (2): 215-9. DOI: 10.1016/j.cgh.2007.11.011. 